\title{
EDITORIAL
}

\section{Synthetic Androgen and New Psycho-Active Compounds (Smart Drugs) Abuse and Dependance. Neuropathological and Toxicological Findings, with an Approach Designed For the Future}

\begin{abstract}
Aims \& Scope
Self-administration of high doses of synthetic androgen (SA) is widespread among young people, to enhance physical aspect and gain muscle mass. The mechanisms of SA toxicity are not yet completely understood while the adverse effects of SA are known to be complex and likely to arise from effects on several organ systems in humans. Moreover, the negative health consequences of SA have many variable as the sex, dose and the duration of administration and many of the side effects may be reversible.

The genomic action of Androgenic Receptors is modulated by a large variety of coregulators, which are proteins that target gene expression by enhancing (coactivator) or restraining (corepressor) transcription. SA may also have direct rewarding or hedonic properties, mediated not so much by their genomic effects (although these may well contribute) but more directly by the effects of SA and their metabolites on plasma membranes.
\end{abstract}

As for other steroids, non-genomic androgen effects typically involve the fast induction of conventional second messenger signal transduction cascades, including increases in cytosolic calcium and activation of protein kinase $\mathrm{A}$, protein kinase $\mathrm{C}$, and MAPK (mitogen- activated protein kinase), leading to diverse cellular effects including smooth muscle relaxation, neuromuscular and junctional signal transmission and neuronal plasticity. Most nongenomic effects involve a membrane receptor, and putative binding sites are described for androgens. The use of AAS among young people has been associated with abuse of additional substances in several cross-sectional survey studies.

More often new psychoactive substances that comprise of natural vegetable and synthetic compounds containing both active ingredients such as caffeine, taurine, ephedrine - essentially stimulating substances - and hallucinogenic substances are frequently used in association with SA especially in non athletes frequenting fitness or sport centers.

Recently, all over the world the availability of new products sold as "Legal Highs" or "Herbal Highs" (psychoactive compounds not included in the list of Controlled Substances) has been described. This new heterogeneous class of products is also named "Smart Drugs" (SD) and includes several types of preparations such as teas, herbal mixtures, incenses, ambient scents, energetic drinks and food additives. Because of the lack of legal regulations to their marketing, SDs are easily available in common shops such as herbalist shops, in the so- called "Smart Shops" or through e-commerce. It is widely reported that the use of SDs is associated with dissociate mental states and mild psychedelic sensations. Moreover, SDs may induce amphetamine-like effects on both fatigueand mood as well as performance enhancement.

This special issue is devoted to the advancement of research in these areas, with particular attention to genetic polymorphisms involved, at the cellular and sub-cellular trafficking, as well as the definition in the diagnostic and pathological changes.

Cognitive enhancers and AASs can be considered as the two sides of the same coin. Both of them represent the more general desire of "human enhancement" which can be defined as the wish to overcome, temporarily or permanently, the existing limits in the human body with natural or artificial methods. With these introduction Busardò et al., discussed the topic of cosmetic neurology, that is a controversial but emerging field, where new psychoactive substances are used to enhance neurological functions [1].

New psychoactive substances (NPS) have completely modified the drug scene and the current landscape of addiction. Synthetic cathinones and synthetic cannabinoids represent more than two thirds of the NPS available in this new drug market. So, Karila and collaborators stated that major health issues have emerged in relation to the somatic, mental, and addictive consequences of their use with persistent unknowns for the future. It is mandatory to develop clinical research and improve management of addiction and poisonings attributed to these NPS [2].

Similarly, Karch reports that among hundreds of cathinones that have been synthesized, or can be synthesized, three molecules in particular appear to account for the content of most products sold as "bath salts." Today the three most commonly encountered are mephedrone, methylone, and MDPV ("bath salts"). Toxicity, most often manifested as psychosis or even fatal excited delirium, seems to be dose related, and more likely to occur in chronic drug abusers [3].

Romolo and collaborators offered a comprehensive review of the scientific literature and internet sites regarding tryptamines and their derivatives, providing a summary of all the available information about the structure of these compounds, their effects in relationship with the routes of administration and their toxicity, including articles reporting cases of 
death related to intake of these substances and analytical methods for the determination and quantification of some tryptamines [4].

The illicit recreational drug of abuse $\gamma$-hydroxybutyrate (GHB), a potent central nervous system depressant depressant, is often encountered during forensic investigations of living and deceased persons. Reliable information about the popularity and extent of GHB use as a recreational drug in countries around the world and its diffusion (including GHB analogous/precursors) in the general population is not easy to find in the literature. Wayne Jones and Busardò, in this respect, stressed that GHB and its analogues continue to represent a drug abuse problem for society indeed, GHB is commonly encountered in forensic investigations and in post-mortem toxicology when poisoning deaths are investigated [5].

Aromatario and collaborators presented a comprehensive review about caffeine that, like other psychoactive substances whose diffusion is regulated by drug legislation, can induce abuse and dependence. Moreover, caffeine, like alcohol and tobacco, is legally used, but, unlike the latter, there is no control or restriction of its sale in the form of high concentration drinks or tablets. Coffee and caffeine-containing products are extremely numerous. They have several effects on the heart and brain, thus it is of interest to examine whether these effects could be detrimental for health [6].

Parr and Joseph presented a review that focuses on the steroidal findings of androgens in dietary supplements. These authors concluded that the large number of designer steroid findings in dietary supplements and the detection of new compounds combined with legal loopholes for their distribution in many countries show that stricter regulations and better information policy are needed in this respect [7].

The aim of the review presented by Sani et al., ii, was to investigate the relationship between AAS use and psychopathology in athletes and identify possible preventive and treatment methods. Exogenously administered testosterone and its synthetic analogs may alter the developmental trajectory of the brain in adolescents and young adults its pattern of adaptation to environmental stimuli. AASs have numerous central nervous system effects, the extent of which varies with several factors, such as the athlete's background resilience, the duration of AAS use and dose, concurrent organic diseases, and use of other medications, alcohol or illegal substances [8].

Frati et al., reviewed the impact of nandrolone decanoate on the Central Nervous System. The purpose of this review was to summarize the literature concerning studies dealing with ND exposure on animal models, mostly rats that mimic human abuse systems (i.e. supraphysiological doses) [9].

Turillazzi and collaborators stated that according to literature, there is a wide scenario of AAS side effects which affect, mainly, the cardiovascular system, the liver, the kidney, the musculoskeletal and the endocrine systems. In the past years, it has become more and more evident that AAS abuse may be detrimental to brain function. In fact, the complexity of gonadal steroid hormone actions is reflected in their broad and diverse effects on a host of integrated systems including reproductive physiology, sexual behavior, stress responses, immune function, cognition, and neural protection [10].

Finally, Fineschi et al., reviewed the so called anabolic androgenic steroid related-deaths due to AAS abuse, trying to evaluate the autoptic, histopathological and toxicological findings in order to investigate the pathophysiological mechanism that underlines this type of death, which is still obscure in several aspects. The review of the literature allowed us to identify 19 fatal cases between 1990 and 2012, in which the autopsy excluded in all cases extracardiac causes of death [11].

In this special issue, researchers discussed clinical, pharmacological, and toxicological aspects of relevance for advancing in these particular topics. Studies dedicated to advanced techniques, such as genomics and proteomics, will provide systematic reviews of border areas and about new horizons opened in doping research, to understand and explain clinical events of binding topical.

\section{REFERENCES}

[1] Frati, P.; Kiryakou, C.; Del Rio, A.; Marinelli, E.; Montanari Vergallo, G.; Zaami, S.; Busardò, F.P. Smart drugs and synthetic androgens for cognitive and physical enhancement: revolving doors of cosmetic neurology. Curr. Neuropharmacol., 2015, 13, 5-11.

[2] Karila, L.; Megarbane, B.; Cottencin, O.; Lejoyeux, M. Synthetic cathinones: a new public health problem. Curr. Neuropharmacol., 2015, 13, 12-20.

[3] Karch, S.B. Cathinone Neurotoxicity ("The "3Ms"). Curr. Neuropharmacol., 2015, 13, 21-25.

[4] Tittarelli, R.; Mannocchi, G.; Pantano, F.; Romolo, F.S. Recreational use, analysis and toxicity of tryptamines. Curr. Neuropharmacol., 2015, 13, 2646.

[5] Busardo, F.P.; Jones, A.J. GHB pharmacology and toxicology: Acute intoxication, concentrations in blood and urine in forensic cases and treatment of the withdrawal syndrome. Curr. Neuropharmacol., 2015, 13, 47-70.

[6] Cappelletti, S.; Piacentino, D.; Sani, G.; Aromatario, M. Caffeine: cognitive and physical performance enhancer or psychoactive drug? Curr. Neuropharmacol., 2015, 13, 71-88.

[7] Joseph, J.F.; Parr; M.K. Synthetic Androgens As Designer Supplements. Curr. Neuropharmacol., 2015, 13, 89-100.

[8] Piacentino, D.; Kotzalidis, G.D.; del Casale, A.; Aromatario, M.; Pomara, C.; Girardi, P.; Sani, G. Anabolic-androgenic steroid use and psychopathology in athletes. A systematic review. Curr. Neuropharmacol., 2015, 13, 101-121.

[9] Busardò, F.P.; Frati, P.; Di Sanzo, M.; Napoletano, S.; Pinchi, E.; Zaami, S.; Fineschi, V. The Impact of Nandrolone Decanoate on the Central Nervous System. Curr. Neuropharmacol., 2015, 13, 122-131. 
[10] Pomara, C.; Neri, M.; Bello, S.; Fiore, C.; Riezzo, I.; Turillazzi, E. Neurotoxicity by Synthetic Androgen Steroids: Oxidative Stress, Apoptosis, and Neuropathology. A review of the literature. Curr. Neuropharmacol., 2015, 13, 132-145.

[11] Frati, P.; Busardò, F.P.; Cipolloni, L.; De Dominicis, E.; Fineschi, V. Anabolic androgenic steroid (AAS) related deaths: autoptic, histopathological and toxicological findings. Curr. Neuropharmacol., 2015, 13, 146-159.

\section{Vittorio Fineschi \\ (Guest Editor)}

Department of Anatomical, Histological

Forensic and Orthopaedic Sciences

Sapienza University of Rome

Viale Regina Elena 336

00161 Rome

Italy

Tel: +390649912722

Fax: +39 064455335

E-mail: vfinesc@tin.it 